Article original

\title{
Apport de la chirurgie endoscopique dans le traitement de
}

\section{l'hydrocéphalie pédiatrique}

\section{Contribution of endoscopic surgery in the treatment of pediatric hydrocephalus}

\author{
Souad DAOUD - Mammar BOUCHAKOUR
}

Service de neurochirurgie $\mathrm{CHU}$ d'Oran

\section{MOTS CLÉS \\ Ventriculocisternostomie - coagulation des plexus choroïde - hydrocéphalie - pédiatrie.}

\section{Résumé}

Introduction - Les dérivations internes par implantation de shunts ont été pour de nombreuses années le traitement quasiment unique de l'hydrocéphalie mais aussi l'objet de nombreuses complications ce qui a poussé le neurochirurgien à développer la chirurgie endoscopique notamment la ventriculocisternostomie et la coagulation des plexus choroïdes.

Matériel et méthode - L'objectif de notre travail est de rapporter l'expérience du service de neurochirurgie du CHU d'Oran dans le traitement de l'hydrocéphalie pédiatrique par chirurgie endoscopique, à travers une étude rétrospective, s'étalant du 1er octobre 2015 au 30 septembre 2016.

Resultats - Nous avons réalisé 67 procédures endoscopiques chez 63 patients âgés de moins de 15 ans dont quatre ont été opérés à deux reprises. L'âge moyen de nos patients était de 20,38 +/- 3,6 mois avec une légère prédominance féminine (3 filles pour 2 garçons) et $75 \%$ de malformations. Nous avons recensé 17 complications dans le post opératoire immédiat, représentées par les crises convulsives (15\%), les collections sous durales (6\%), les fistules du liquide céphalorachidien à travers la cicatrice du cuir chevelu (3\%) et les complications infectieuses (1\%). Le taux de succès du traitement endoscopique de l'hydrocéphalie dans notre série était de $74,62 \%$. Nous avons déploré deux décès (3\%).

Conclusion - La chirurgie endoscopique présente peu de complications. Elle représente une alternative efficace pour le traitement de l'hydrocéphalie pédiatrique. 


\section{KEY WORDS}

Endoscopic Third
Ventriculostomy -
coagulation of choroid
plexuses hydrocepha-
lus - pediatric patients.

\section{Introduction}

L'hydrocéphalie (HDC) est une dilatation active des ventricules cérébraux liée à un trouble de la circulation et/ ou de la résorption du liquide céphalo-rachidien (LCR). C'est l'une des pathologies neurologiques les plus fréquentes en neurochirurgie pédiatrique [1]. Son incidence est variable en fonction du niveau socioéconomique du pays considéré [2]. Les dérivations ventriculo-péritonéales (DVP) ont été pour de nombreuses années, le seul traitement de l'hydrocéphalie mais aussi l'objet de complications infectieuses dans 0,5\% à $10 \%$ des cas [3] et mécaniques dans $50 \%$ à $100 \%$ des cas [ 4 , $5,6,7]$.

Ces complications ont incité les neurochirurgiens à développer la chirurgie endoscopique notamment la ventriculocisternostomie (VCS) et la coagulation des plexus choroïdes (CPC). Bien qu'elle soit connue depuis le début du 20ème siècle, cette technique n'a prouvé son efficacité qu'au cours de ces dernières années, grâce aux progrès considérables dans l'amélioration de la résolution du matériel endoscopique [8]. La VCS a pour but, de créer une voie de contournement de l'obstacle. Elle consiste à mettre en communication le troisième ventricule et les espaces sous-arachnoïdiens au niveau de la citerne inter pédonculaire par perforation du plancher ventriculaire sous endoscope. II s'agit d'un traitement qui rétablit une circulation du LCR proche de la normale et évite ainsi les complications mécaniques et infectieuses de la mise en place de corps étranger (shunt).

L'objectif de ce travail est de rapporter l'expérience du service de Neurochirurgie du CHU d'Oran dans le traitement de l'hydrocéphalie pédiatrique par chirurgie endoscopique.

\section{Matériels et méthodes}

Il s'agit d'une d'étude descriptive, rétrospective, mono centrique, menée au service de neurochirurgie du $\mathrm{CHU}$ d'Oran entre octobre 2015 et septembre 2016.

Notre étude a porté sur des cas pédiatriques d'hydrocéphalie traités par chirurgie endoscopique. Nous n'avons pas inclus les patients présentant une dilatation ventriculaire passive ou secondaire à une atrophie cérébrale. Nous avons étudié les paramètres suivants : l'âge, le sexe, les signes cliniques, les étiologies, les données neuroradiologiques, les complications postopératoires, et le taux de dysfonctionnement de la VCS. Les causes de l'hydrocéphalie ont été classifiées comme suit :

- Congénitales en rapport avec différentes malformations de l'enfant et en particulier (la sténose de l'aqueduc de Sylvius, des dysraphismes, la malformation de DANDY WALKER) ; 
- Tumorales : dominée par les tumeurs de la fosse cérébrale postérieure ;

- Infectieuses : post-méningite ;

- Post-hémorragiques (hémorragie des prématurés par exemple).

Nous avons considéré comme « complications post-opératoires », les fuites de LCR par la cicatrice, les méningites, les ventriculites, les collections sous-durales, ainsi que les crises d'épilepsie.

Dans notre étude, le succès de la VCS a été défini par la régression complète et durable de la symptomatologie clinique en rapport avec l'hydrocéphalie, avec l'absence de toute autre cure chirurgicale de l'hydrocéphalie après la réalisation de la VCS.

\section{Technique chirurgicale}

Nous avons utilisé un neuro-endoscope rigide sans bras articulé type WALF.

L'intervention est réalisée sous anesthésie générale, le patient installé en décubitus dorsal, tête maintenue dans l'axe du corps, antéfléchie de 30 à $45^{\circ}$. L'endoscope est introduit dans le ventricule latéral puis le trou de Monro est chargé pour aborder le troisième ventricule.

La perforation du plancher du troisième ventricule est réalisée juste en arrière du clivus, sur la ligne médiane entre l'infundibulum et les corps mamillaires (Figure $1: a$ et b). La stomie est ensuite agrandie en utilisant un cathéter à ballonnet de type Fogarty $n^{\circ} 4$, gonflé pendant une trentaine de secondes (Figure :1c).

Figure 1 : perforation du plancher du $3 e$ ventricule sous endoscope
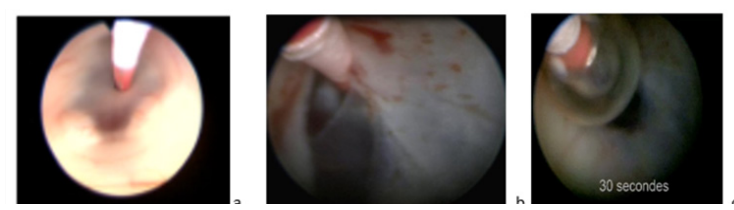

La perméabilité de la stomie est vérifiée en introduisant l'optique jusqu'à ses berges afin de rechercher « la membrane résiduelle de Lillequiste » (Figure : 2 ).

Figure 2 : au delà du plancher du troisième ventricule

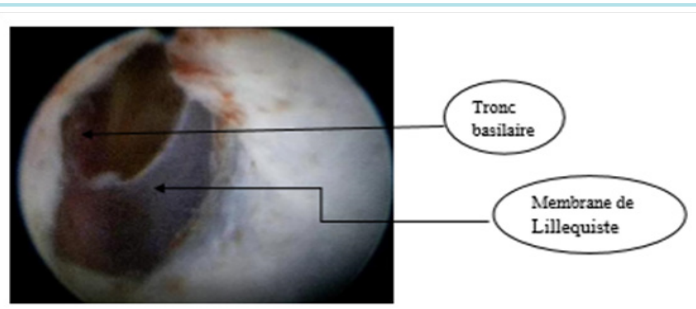

Pour la CPC, le plexus choroïde du ventricule latéral est complètement cautérisé à l'aide d'un courant de coagulation mono polaire ou bipolaire à basse tension dans un but de réduire la sécrétion du LCR.

Analyses statistiques

Les données ont été saisies sur le logiciel EPI info version 3.5.3. La fréquence a été exprimée en pourcentage. Le degré de signification a été mesuré par le test $\mathrm{x} 2$ de Pearson et le $x 2$ corrigé de Yates pour un risque de première espèce fixé à $5 \%$.

\section{Résultats}

Durant la période d'étude qui s'est étalée du 1er octobre 2015 au 30 septembre 2016, nous avons réalisé 67 gestes de ventriculocisternostomie chez 63 cas consécutifs, dont quatre ont été opéré à deux reprises. L'âge moyen de nos patients était de 20,38+/- 3,6 mois avec des extrêmes allant de 4 jours à 15 ans. Nous avons constaté une légère prédominance féminine avec un sexe ratio estimé à 1,5 (Tableau 1).

Tableau 1. Répartition des cas selon le sexe et les tranches d'âge

\begin{tabular}{|c|c|}
\hline & Nombre de cas \\
\hline PATIENTS & $67(100 \%)$ \\
\hline Filles & 40 \\
\hline Garçons & 27 \\
\hline AGE & $04(6 \%)$ \\
\hline$<1$ mois & $40(59,7 \%)$ \\
\hline 1 à 6 mois & $12(17,9 \%)$ \\
\hline 6 à 18 mois & $11(16,4 \%)$ \\
\hline$>2$ ans & \\
\hline
\end{tabular}

Le mode de révélation clinique le plus fréquent était la macrocrânie (augmentation du périmètre crânien +2 DS) avec une fréquence de plus de $60 \%$. Chez les enfants de plus de 2 ans, la présentation la plus fréquente était le syndrome d'hypertension intracrânienne dans $16 \%$.

Dans notre série, les causes malformatives viennent en tête des causes de l'hydrocéphalie (50 patients soit $75 \%$ ), suivies des causes tumorales (13 patients soit 19\%). Viennent ensuite les causes infectieuses $(4,5 \%)$ et post hémorragiques $(1,5 \%)$. Les causes malformatives touchent principalement les patients âgés de moins de 2 ans.

Les lésions tumorales sont rencontrées surtout chez les patients âgés de plus de 2 ans et touchent principalement la fosse cérébrale postérieure (Figure 3). 
Les myéloméningocèles sont en tête des causes malformatives de l'hydrocéphalie avec un taux de $58 \%$, suivies de la sténose de l'aqueduc de Sylvius avec un taux de $28 \%$ et en dernier la malformation de dandy Walker avec un taux de 14\% (Figure 3).

Afin de mettre en évidence le type et l'étiologie de l'hydrocéphalie, un scanner cérébral avec et sans injection de produit de contraste a été réalisé chez la majorité de nos patients (66\%).

L'IRM cérébrale a été réalisée chez seulement 34\% des cas. Elle permet une meilleur analyse des citernes de la base en particulier la citerne inter pédonculaire, siège de la réalisation de la VCS.

Au cours de notre expérience, plusieurs difficultés techniques ont été rencontrées en per opératoire, dont la plus fréquente était la qualité du LCR (trouble dans 12\% des cas ou hémorragique dans $11 \%$ des cas) rendant la vision impos- sible. Pour palier à cette difficulté, nous avons procédé au rinçage abondant au sérum salé chauffé à $37^{\circ} \mathrm{C}$.

Le taux de succès global de la VCS était de 74,62 \% (50 procédures sur 67$)$. Les dix-sept échecs de la procédure sont survenus pendant les six premiers mois. Pour les patients âgés de plus de deux ans, le taux de succès était de $100 \%$. Pour ceux âgés de 1 à 18 mois, le taux de succès était plus important que le taux d'échec presque le double ; alors que pour la tranche d'âge de moins d'un mois le taux d'échec était significativement plus important que le taux de succès $(p<10-5)$ (Tableau 2).

Concernant le sexe, le taux de succès était plus élevé chez les filles $(p<0,02)$.

Des taux de succès plus importants ont été observés dans les hydrocéphalies congénitales par sténose de l'aqueduc de Sylvius et les hydrocéphalies acquises secondaires à une lésion tumorale alors que pour les hydrocéphalies post méningitiques (taux de succès $<40 \%$ ) ou post hémorragiques (taux de succès $<3 \%$ ) les résultats étaient décourageants $(p<10-6)$

Figure 3 : répartition des causes d'hydrocéphalie dans notre série

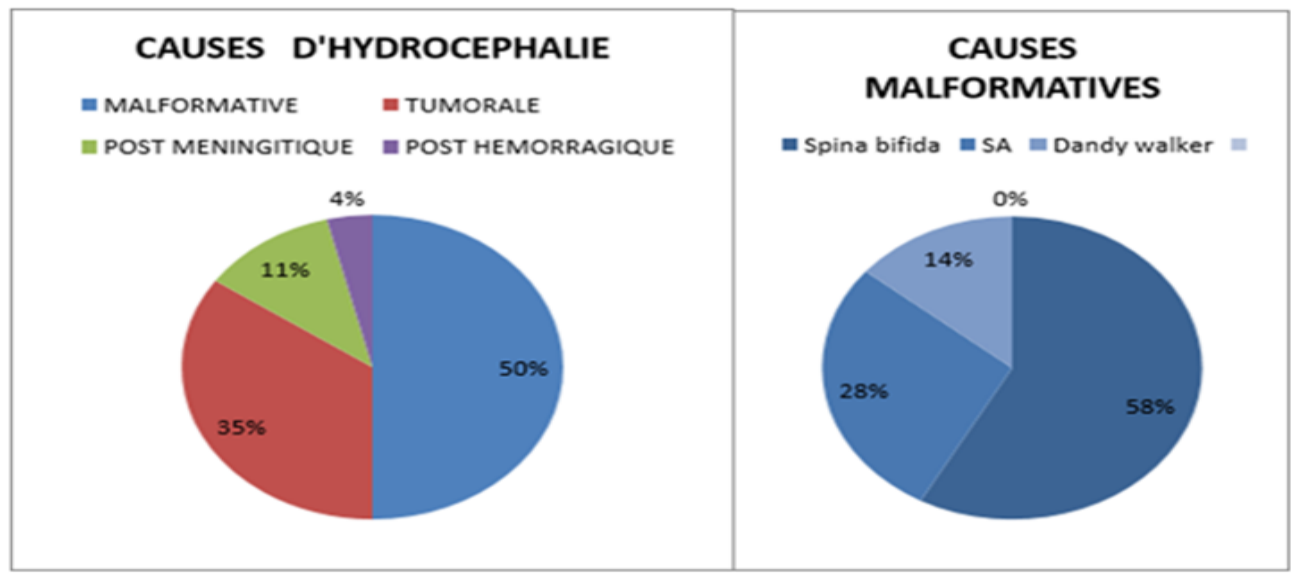

$\mathrm{SA}=$ sténose de 1'aqueduc de Sylvius

Tableau 2 : répartition des résultats en fonction de l’âge du sexe

\begin{tabular}{llllll}
\hline & succès & $\%$ & échec & $\%$ & $\mathbf{p}$ \\
\hline âge & & & & & $<0.00001$ \\
\hline$<$ lmois & 1 & $1 \%$ & 3 & $4 \%$ & \\
\hline $\mathbf{1 - 6}$ mois & 28 & $42 \%$ & 12 & $18 \%$ & \\
\hline $\mathbf{6 - 1 8}$ mois & 10 & $15 \%$ & 2 & $3 \%$ & \\
\hline$>\mathbf{2}$ ans & 11 & $16 \%$ & 0 & $0 \%$ & \\
\hline sexe & & & & & $<0.02$ \\
\hline filles & 34 & $51 \%$ & 6 & $9 \%$ & \\
\hline garçon & 16 & $24 \%$ & 11 & $16 \%$ & \\
\hline
\end{tabular}


Concernant les examens neuroradiologiques, nous avons noté que les indications de la VCS posées suite à la réalisation d'une IRM cérébrale, donnaient de meilleurs résultats que celles posées après scanner $(p<0,01)$.

Nous avons recensé 17 complications (25\%) dans le post opératoire immédiat, dont les plus fréquentes sont les crises convulsives retrouvées chez 10 cas (15\%) suivies de la collection sous-durale chez 4 cas (6\%), et des fistules du LCR à travers la cicatrice du cuir chevelu chez deux patients (3\%). Nous avons enregistré une seule complication infectieuse. Le taux de décès dû à la procédure était de $3 \%(2 / 67)$.

\section{Discussion}

Notre série comprend 63 enfants dont l'âge est inférieur ou égal à 15 ans, sur une période d'une année. Le nombre de cas des études varie selon les centres. Ainsi, dans les séries publiées on trouve l'étude de DECQ et al [8], qui porte sur 38 cas ; Gorayeb [9] a rapporté une série de 36 patients de moins d'un an. La plus grande série est celle de Warf qui a porté sur 300 enfants [10,11].

Dans notre étude, la tranche d'âge de 1 à 18 mois était la plus représentée, avec un taux de succès se rapprochant du taux d'échec ; alors que pour les moins d'un mois le taux d'échec était plus important. Ce taux était nul chez les enfants de plus de deux ans. Certains auteurs, rapportent un taux de réussite de VCS à $40 \%$ lorsque les enfants sont opérés à l'âge de deux ans, et à $71 \%$ lorsqu'ils sont opérés plus tard dans l'enfance ou l'adolescence [12,13]. Dans l'étude de Grunert et al, le taux de réussite était significativement plus faible chez les nourrissons (22,3\%) par rapport aux enfants de plus d'un an $(71,4 \%)$ et aux adultes $(81,6 \%)[14,15]$. Par ailleurs, Kim et al, ont identifié l'âge comme facteur prédictif de succès de la VCS [16]. Dans la littérature, il existe une légère prédominance du sexe masculin [17]. Cette prédominance s'explique en partie par le fait que l'hydrocéphalie congénitale peut se transmettre selon un mode récessif lié à l'X $[18,19]$. Dans notre série, il y avait une prédominance féminine avant l'âge de 6 mois. Cela peut être expliqué par le nombre de filles plus élevé dans notre série.

Les causes de l'hydrocéphalie varient en fonction de l'âge (congénitales ou malformatives chez les nouveau-nés et nourrissons, et tumorales chez les grands enfants et les adultes). Chez nos patients, les causes les plus fréquentes d'hydrocéphalie étaient les malformations cérébrales, représentées essentiellement par les sténoses congénitales de l'aqueduc de Sylvius, les spina bifida et la malformation de dandy Walker. Nous pouvons attribuer ceci au fait que notre étude a concerné une population pédiatrique.
Le taux de succès du traitement endoscopique de ce type d'hydrocéphalie dépend du type de la malformation, les meilleurs résultats dans notre série ont été obtenus dans les sténoses de l'aqueduc de Sylvius avec un taux de succès de $92 \%$.

Dans la littérature, les taux de réussite de la VCS chez les nourrissons atteints de sténose congénitale de l'aqueduc de Sylvius sont toutefois inférieurs à ceux rapportés chez les enfants plus âgés et les adultes présentant une sténose aqueducale acquise [20, 21, 22].

Des taux d'échec allant jusqu'à 50\% sont rapportés chez les patients atteints de myéloméningocéle [23, 24,25]. Cependant, dans la série de Warf et al, le taux de succès était plus important $(78 \%)$ chez des nourrissons atteints d'hydrocéphalie associée à une myéloméningocéle [26,27].

Pour les malformations de Dandy-Walker, nous avons recensé 7 cas pour lesquels nous avons enregistré un taux de réussite de $57 \%$. Dans la littérature, les données concernant cette malformation sont contradictoires [18, 19, 28,29, 30].

Pour les hydrocéphalies post méningitiques, nous avons enregistré trois cas, avec un taux de réussite de 5,7\%. Warf et al, ont rapporté les résultats d'une série de patients pédiatriques avec l'hydrocéphalie post infectieuse. Les taux de réussite chez ces patients étaient plus faibles que chez les patients atteints d'hydrocéphalie due à une sténose de l'aqueduc de Sylvius d'origine non infectieuse $[10,11]$. Nous avons enregistré un seul cas d'hydrocephalie post hémorragique pour lequel nous avons signale un échec du traitement endoscopique. Cet échec pourrait être expliqué par l'obstruction des citernes basales et des villosités arachnoïdiennes, en plus de l'aqueduc de Sylvius [31, 32, 33].

Dans notre étude, le taux de réussite du traitement endoscopique était de $100 \%$ dans les hydrocéphalies secondaires à une tumeur cérébrale qui bloque la circulation du LCR. Dans la littérature ce taux est supérieur à 75\% [28].

Idéalement, avant chaque VCS, une IRM cérébrale doit être réalisée [34]. Elle joue un rôle capital dans le diagnostic étiologique et topographique. A cette situation «idéale » s'opposent les contraintes liées à la disponibilité et au cout de cet examen. Dans notre série, l'indication de la VCS a été retenue après la réalisation d'un scanner cérébral seul dans $66 \%$ des cas.

Dans la littérature les complications infectieuses de la VCS varient de 1 à $5 \%[35,36,37]$ représentées essentiellement par les infections de la cicatrice opératoire, les ventriculites et les méningites. Dans notre étude, ce taux était estimé à $3 \%$. De même, l'incidence des collections sous durales post opératoires dans notre série $(6 \%)$ rejoint celle rapportée 
dans la littérature $(2 \%)[28,36,38]$.

Nous avons enregistré 10 cas de comitialité post chirurgie endoscopique. Une comitialité post VCS est également décrite dans la littérature mais elle est rare et résulte le plus souvent d'une altération de l'état général préexistante [39].

Dans notre étude, quatre patients ont été réopérés (révision de la VCS). Les obstructions à distance de la VCS sont habituellement secondaires à la formation d'un tissu cicatriciel épendymaire $[40,41]$. La formation d'une nouvelle membrane arachnoïdienne dans la citerne inter pédonculaire a également été décrite [42].

\section{Conclusion}

Dans toutes les spécialités chirurgicales il y’a actuellement une tendance à rechercher des méthodes mini invasives qui permettent de réduire les complications postopératoires et améliorer le résultat.

Notre étude confirme l'intérêt de la VCS comme stratégie alternative efficace pour le placement de shunt dans le traitement de l'hydrocephalie obstructive chez l'enfant.

Des études multicentriques prospectives portant sur de grandes séries sont indispensables afin de valider nos résultats.

\section{Conflits d'intérêt}

Les auteurs déclarent n'avoir aucun conflit d'intérêt.

\section{Références bibliographiques}

[1]. SAINTE-ROSE C. Hydrocéphalie de l'enfant. Neurochirurgie Clin Perinatol 1997; $1:$ 589-605

[2]. LABCHIR. N. Prise en charge de l'hydrocéphalie de l'enfant de moins de 15 ans à

l'Hôpital Mohamed V de MEKNES. Thèse de médecine Casablanca, $2002 ; 02$

[3]. RENIER D., LACOMBE J ., PIERRE-KHAN A . et all « facteurs causing acute shunt infection. Computer analysis of 1174 operations », J. Neurosurg.61,p. 10721078,1984

[4]. BIERBRAUER K.S.,STORRS B.B.,MCLONE D.G et all «A prospective ,randomized study of shunt function and infection as a function of shunt placement », pediatr.Neurosurg.91,p.287-291.1990-91.
[5]. COLLINS P.,HOKLEY AD.,WOOLLAM D.H.M., « surface ultra sructure of tissues occluding ventricular catheter » J.N eurosurg.48, p,609-613,1978.

[6]. GOWER D,J ., LEWIS J.C.,KELLY D.L .JR., « sterile shunt malfunction. Ascanning electron microscopic perspective "J.Neurosurg.62,p.1079-1084,1984.

[7]. SAINTE- ROSE C., « shunt obstructive :Apreventable complication? » pediatr.Neurosurg.19,p.156-164.1993

[8]. DECQ. Ph L'endoscopie en neurochirurgie : le neuroendoscope d'après DECQ , 1998.

[9]. GORAYEB RP; CAVALHEIRO S., ZYMBERG SY. Endoscopic third ventriculostomy in children younger than 1 year of age. J Neurosurg (Pediatrics 5) 2004; 100: 427-429

[10]. Warf BC. Hydrocephalus in Uganda: The predominance of infectious origin and primary management with endoscopic third ventriculostomy. J Neurosurg 2005;102(1 Suppl):1-15.

[11]. Warf BC, Dagi AR, Kaaya BN, Schiff SJ. Five-year survival and outcome of treatment for postinfectious hydrocephalus in Ugandan infants. J Neurosurg Pediatr 2011;8:502-8

[12]. JONES RFC., KWOK BCT., STENING WA., VONAU M. The current status of endoçcopic third ventriculostomy in the management of non-communicating hydrocephalus.Minim Invasive Neurosurg 1994; 37: $28-36$

[13]. JONES RFC., STENING WA., BRYDON M. Endoscopic third ventriculostomy. Neurosurgery 1990; 26:86-92

[14]. GUTNER P.,BASS T., GUDEMAN S.K et all , « surgical management of post hemorrhagic hydrocephalus in 22 low-birth-weight infants » childs erv.syst.8.p.198202,1992.

[15]. HOPF NJ., GRUNERT P., FRIES G., RESCH KDM., PERNECZKY A. Endoscopic third ventriculostomy: outcome analysis of 100 consecutive procedures.

Neurosurgery 1999; 44: 795-806

[16]. Kim SK, Wang KC, Cho BK. Surgical outcome of pediatric hydrocephalus treated by endoscopic III ventriculostomy: prognostic factors and interpretation of postoperative neu- roimaging. Childs Nerv Syst 2000;16:161-69.

[17]. ARTHUR E., LYONS M. D. Hydrocephalus first illustrated.Neurosurg.

1995. 37. : 511-513.

[18]. RODRIGUEZ CRIADO G., PEREZ AYTES A., MARTINEZ F., VOS Y.J. VERLIND E., GONZALEA., et all. X-linked hydrocephalus: another two families with an L1 mutation.Genet Couns. 2003, 14(1):57-65.

[19]. SCHRANDER-STUMMPEL C.T., VOS Y.J. From gene to disease; $\mathrm{X}$-linked hydrocephalus and LiCAM. 
[20] . Elgamal EA, El-Dawlatly AA, Murshid WR, El-Watidy SM, Jamjoom ZA. Endoscopic third ventriculostomy for hydrocephalus in children younger than 1 year of age. Childs Nerv Syst 2011;27:111-6.

[21]. BEEMS T., GROTENHUIS JA. Long-term complications and definition of failures of neuroendoscopic procedures Child's Nerv Syst 2004; 20: 868-877

[22]. Singh D, Gupta V, Goyal A, Singh H, Sinha S, Singh AK, et al. Endoscopic third ventriculostomy in obstructed hydrocephalus. Neurol India 2003;51:39-42

[23]. Warf BC, Campbell JW, Riddle E. Initial experience with combined endoscopic third ventriculostomy and choroid plexus cauterization for post-hemorrhagic hydrocephalus of prematurity: The importance of prepontine cistern status and the predictive value of FIESTA MRI imaging. Childs Nerv Syst 2011;27:1063-71.

[24]. Warf BC Comparison of endoscopic third ventriculostomy alone and combined with choroid plexus cauterization in infants younger than 1 year of age: a prospective study in 550 African children. J Neurosurg 2005. 103(6 Suppl): 475-481.

[25]. Warf BC .Congenital idiopathic hydrocephalus of infancy: the results of treatment by endoscopic third ventriculostomy with or without choroid plexus cauterization and suggestions for how it works. Childs Nerv Syst 2013 . 29(6): 935-940.

[26]. Warf BC, Campbell JW. Combined endoscopic third ventriculostomy and choroid plexus cauterization as primary treatment of hydrocephalus for infants with myelomeningocoele: Long term results of a prospective intent-to-treat study in 115 East African infants. J Neurosurg Pediatr 2008;2:310-6.

[27]. Warf BC, Dewan M, Mugamba J Management of Dandy-Walker complex-associated infant hydrocephalus by combined endoscopic third ventriculostomy and choroid plexus cauterization. J Neurosurg Pediatr 2011. 8(4): 377-383.

[28]. Sebastien freppel .la ventriculocisternostomie dans le traitement de l'hydrocéphalie étude rétrospective de 68patients ; thèse de médecine Nancy2006

[29]. Garg A, Suri A, Chandra PS, Kumar R, Sharma BS, Mahapatra AK. Endoscopic third ventriculostomy: 5 years'experience at the All India Institute of Medical Sciences. Pediatr Neurosurg $2009 ; 45 ; 1$-5.

[30]. Mohanty A, Biswas A, Satish S, Praharaj SS, Sastry KV. Treatment options for Dandy-Walker malformation. J Neurosurg 2006;105(5 Suppl):348-56.

[31]. Fukuhara T, Shimizu T, Namba Y. Limited efficacy of endoscopic third ventriculostomy for hydrocephalus following aneurysmal subarachnoid hemorrhage. Neurol Med Chir (Tokyo) 2009;49:449-55.

[32]. WHITEHEAD WE., KESTLE .IRW. The treatment of cerebrospinal fluid shunt infections. Pediatr Neurosurg 2001; 35: 205-21 0

[33]. Rekate HL. Selecting patients for endoscopic third ventriculostomy. Neurosurg Clin N Am 2004;15:39-49.
[34]. CINALLI G., DI ROCCO C., MASSIMI L., SPENNATO P., CIANCIULLI E., TAMBURRINI G. Endoscopic third ventriculostomy in the treatment of hydrocephalus in pediatric patients. Adv Tech Stand 2006; $31: 1$ 19-21 9

[35]. BEEMS T., GROTENHUIS JA. Long term complications and definition of failures of neuroendoscopic procedures Child's Nerv Syst 2004; 20: 868-877

[36]. SCHROEDER HWS., OERTEL J., GAAB MR. Incidence of complications in neuroendoscopic surgery. Childs Nerv Syst 2004; 20: 878883

[37]. Tew jm., van loveren hr., keller jt. Atlas of operative microneurosurgery vol. 2. Brain tumors. W.B. Saunders Company, $2001 / 127$

[38]. GENITORI L., PERETTA P., MUSSA F., GIORDANO F.Endoscopic third ventriculostomy in children: are age and etiology of hydrocephalus predictive factors influencing the outcome in primary and secondary treated patients?

A series of 328 patients and 353 procedures. II CURAC Congress, Munich, 8 octobre 2004

[39]. SIOMIN V., WEINER H., WISOFF J., CINALLI G., PIERRE-KHAN A., SAINTEROSE C.,ABBOTT R., ELRAN H., BENI-ADAN1 L., OUAKINE G., CONSTANTIN1 S. Repeat endoscopic third ventriculostomy: is it worth trying? Childs Nerv Syst 2001; $\quad$ 17: 551-555

[40]. CINALLI G., SAINTE-ROSE C., CHUMAS P., ZERAH M., BRLINELLE F.,LOT G., PIERRE-KHAN A., RENIER D. Failure of third ventriculostomy in the treatment of aqueducal stenosis in children. J Neurosurg 1999; 90: 448-454

[41]. HADER WJ., DRAKE J., COCHRANE D., SPARROW O., JOHNSON ES., KESTLE J. Death after late failure of third ventriculostomy in children. Report of three cases. J Neurosurg 2002; 97: 21 1-215.

[42]. WAGNER W., KOCH D.Mechanisms of failure after endoscopic third ventriculostomy in young infants.J Neurosurg (Pediatrics 1 ) 2005; 103: 43-49

[43]. DECQ.Ph ;Anatomie endoscopique ventriculaire. Morphologie, 20058912 21, Masson paris. 
